TRANSACTIONS OF THE

AMERICAN MATHEMATICAL SOCIETY

Volume 354, Number 9, Pages 3601-3619

S 0002-9947(02)03005-2

Article electronically published on May 7, 2002

\title{
DIFFUSIVE LOGISTIC EQUATION WITH CONSTANT YIELD HARVESTING, I: STEADY STATES
}

\author{
SHOBHA ORUGANTI, JUNPING SHI, AND RATNASINGHAM SHIVAJI
}

\begin{abstract}
We consider a reaction-diffusion equation which models the constant yield harvesting to a spatially heterogeneous population which satisfies a logistic growth. We prove the existence, uniqueness and stability of the maximal steady state solutions under certain conditions, and we also classify all steady state solutions under more restricted conditions. Exact global bifurcation diagrams are obtained in the latter case. Our method is a combination of comparison arguments and bifurcation theory.
\end{abstract}

\section{INTRODUCTION}

We study the nonlinear boundary value problem

$$
\begin{cases}\Delta u+a u-b u^{2}-c h(x)=0, & x \in \Omega, \\ u=0, & x \in \partial \Omega,\end{cases}
$$

where $a, b, c$ are positive constants, $\Omega$ is a smooth bounded region with $\partial \Omega$ in class $C^{2}$ in $\mathbf{R}^{n}$ for $n \geq 1$, and $h: \bar{\Omega} \rightarrow \mathbf{R}$ satisfies $h(x)>0$ for $x \in \Omega, \max _{x \in \bar{\Omega}} h(x)=1$ and $h(x)=0$ for $x \in \partial \Omega$. We assume $h \in C^{\alpha}(\bar{\Omega})$ throughout the paper. A direct consequence is that any solution of (1.1) belongs to the class $C^{2, \alpha}(\bar{\Omega})$.

(1.1) arises from the population biology of one species. Let $u(t, x)$ be the concentration of the species or the population density. We assume that (a) the species disperses randomly in the bounded environment $\Omega$; (b) the reproduction of the species follows the logistic growth; (c) the boundary $\partial \Omega$ of the environment is hostile to the species; and (d) the environment $\Omega$ is homogeneous (i.e., the diffusion does not depend on $x)$. Then it is well known that $u(t, x)$ satisfies the reactiondiffusion equation

$$
\frac{\partial u}{\partial t}=D \Delta u+a u\left(1-\frac{u}{N}\right), \quad(t, x) \in(0, T) \times \Omega,
$$

with the initial and boundary conditions

$$
\begin{aligned}
& u(t, x)=0, \quad(t, x) \in(0, T) \times \partial \Omega, \\
& u(0, x)=u_{0}(x) \geq 0, \quad x \in \Omega,
\end{aligned}
$$

where $D>0$ is the diffusion coefficient, $a>0$ is the linear reproduction rate and $N>0$ is the carrying capacity of the environment. (See Murray $[\mathrm{M}$ for details.)

Received by the editors September 5, 2001 and, in revised form, October 15, 2001.

2000 Mathematics Subject Classification. Primary 35J65; Secondary 35J25, 35B32, 92 D25.

Key words and phrases. Diffusive logistic equation, harvesting, steady states, comparison methods, bifurcation. 
Equation (1.2) is often called Fisher's equation after Fisher $[\mathbf{F}$, and it was also studied by Kolmogoroff, Petrovsky and Piscounoff [KP.

In many ecological systems, harvesting or predation of the species occurs. For example, fishing or hunting of the species $u$ could happen. Hence it is natural to add a harvesting term to the right-hand side of (1.2), and the equation would be

$$
\frac{\partial u}{\partial t}=D \Delta u+a u\left(1-\frac{u}{N}\right)-p(t, x, u)
$$

where $p(t, x, u) \geq 0$ for all possible $(t, x, u)$ values. In this paper, we consider the case of constant yield harvesting (not dependent on the density $u$ or on $t$ ). In particular, we consider the case

$$
p(t, x, u) \equiv \operatorname{ch}(x),
$$

where $c>0$ is a parameter which represents the level of harvesting, $h(x)>0$ for $x \in \Omega, h(x)=0$ for $x \in \partial \Omega$ and $\|h\|_{\infty}=1$. So $\operatorname{ch}(\cdot)$ can be understood as the rate of the harvesting distribution, and the harvesting only occurs in the interior of the environment. Such a harvesting pattern arises naturally from fishery management problems, where $\operatorname{ch}(x)$ is related to the fishing quota imposed by regulating authorities. The equation (1.4) with (1.5) is a generalization of the wellknown ordinary differential equation logistic model with constant yield harvesting (see $[\mathrm{Cl}],[\mathrm{BC}]$ ). With a standard non-dimensionalization process, we can reduce (1.4) to

$$
\frac{\partial u}{\partial t}=\Delta u+a u-b u^{2}-\operatorname{ch}(x),
$$

and the steady state solutions of (1.6) and (1.3) satisfy (1.1).

Mathematically, (1.6) and (1.3) generate a semiflow in the Sobolev space $W_{0}^{1,2}(\Omega)$. When $p(t, x, u) \equiv 0$ (i.e. the logistic case), the dynamics of (1.2) and (1.3) has been completely studied (see Henry [He]). Here we briefly describe the results for the logistic case. We denote by $\lambda_{k}$ the $k$-th eigenvalue of

$$
\begin{cases}\Delta \phi+\lambda \phi=0, & x \in \Omega \\ \phi=0, & x \in \partial \Omega\end{cases}
$$

In particular, $\lambda_{1}>0$ is the principal eigenvalue with a positive eigenfunction $\phi_{1}$ satisfying $\left\|\phi_{1}\right\|_{\infty}=1$.

For (1.2) and (1.3), the following facts have been proved:

1. $v_{0} \equiv 0$ is a steady state solution for any $a, N>0$; when $0<a \leq \lambda_{1}, v_{0}$ is the unique nonnegative steady state solution; and when $a>\lambda_{1}$, there is a unique positive steady state solution $v_{a}$.

2. The set

$$
C_{0}=\left\{\varphi \in W_{0}^{1,2}(\Omega): \varphi(x) \geq 0 \text { on } \Omega\right\}
$$

is positively invariant; for any initial value $u_{0}(\cdot) \in C_{0}$, the solution $u(t, \cdot)$ exists for all $t \in(0, \infty)$ and is uniformly bounded in $W_{0}^{1,2}(\Omega) \cap W^{2,2}(\Omega)$.

3. Let $u_{0}(\cdot) \in C_{0}$. Then when $0<a \leq \lambda_{1},\|u(t, \cdot)\|_{W^{1,2}(\Omega)} \rightarrow 0$ as $t \rightarrow \infty$; when $a>\lambda_{1},\left\|u(t, \cdot)-v_{a}(\cdot)\right\|_{W^{1,2}(\Omega)} \rightarrow 0$ (unless $u_{0} \equiv 0$ ) as $t \rightarrow \infty$.

The results on the steady state solutions are well-known, see for example [BK], [SY], and for the sake of completeness, a proof based on bifurcation theory is given in Section 2.3, Theorem [2.5. The results on the dynamical systems for $\Omega=(0,1)$ 


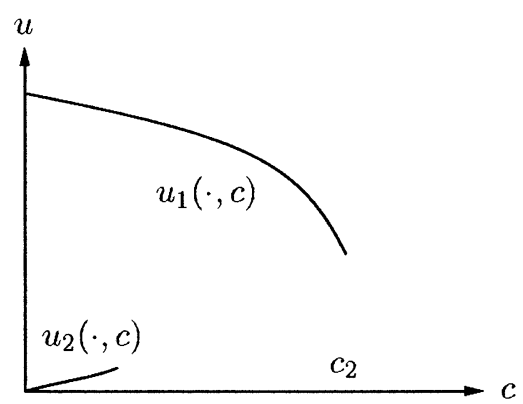

FIGURE 1.

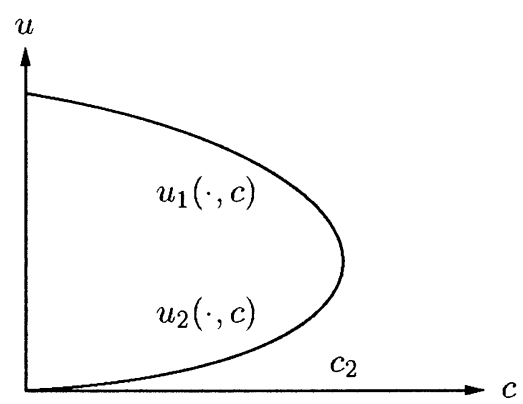

FIGURE 2.

can be found in $[\mathrm{He}$, but the higher-dimensional version can be easily carried over using the same proof.

For the equation with harvesting term $c h(x)$, there is no such perfect structure as in the logistic equation. First, the set $C_{0}$ is not positively invariant, since the nonlinearity $f(x, u)=a u-b u^{2}-c h(x)$ does not satisfy $f(x, 0) \geq 0$, and so the maximum principle does not hold here. Second, the equation has possibly more than one positive steady state solution; one of them is stable, but the attraction basin of the stable steady state solution is not clear.

In this paper and a forthcoming paper we overcome some of these difficulties, and partially describe some important dynamical behavior of the system. In this paper we concentrate on the set of positive steady state solutions, and we shall study the dynamic behavior of the system in the forthcoming paper. When $a \leq \lambda_{1}$, $c>0$, it is easy to show that (1.1) has no nonnegative solutions. When $a>\lambda_{1}$, it becomes more delicate, and we prove the following results:

1. When $0<c \leq c_{1}$, 1.1 has a positive steady state solution $u_{1}$, which is unique in the set

$$
C_{c}=\left\{\varphi \in W_{0}^{1,2}(\Omega): \lambda_{1} \varphi(x) \geq \operatorname{ch}(x) \text { on } \Omega\right\} .
$$

2. When $0<c<c_{2}\left(>c_{1}\right)$, (1.1) has a positive stable steady state solution $u_{1}$, and $u_{1}$ is the maximal steady state solution.

3. When $c>c_{2}$, there is no nonnegative steady state solution. 


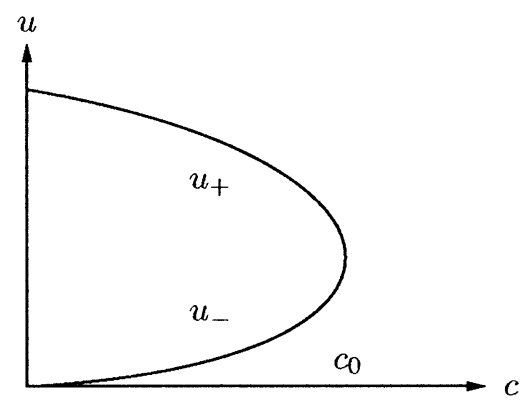

FIGURE 3.

In fact, when $c \in\left(0, c_{1}\right)$, we will show that the unique positive steady state $u_{1}$ also solves the "obstacle" problem $a u_{1}-b u_{1}^{2}-\operatorname{ch}(x)>0$ for $x \in \Omega$. Thus $u_{1}$ is a subharmonic solution as $v_{a}$ (solution of the logistic equation) and represents a biologically meaningful steady state solution for this harvesting case. (See Fig. 1 for illustration. (1.1) may have a second solution for small $c>0$ - see Theorem 3.3 and the Remarks at the end of Section 3.)

The results above hold for any $a>\lambda_{1}$. When $a>\lambda_{1}$ is sufficiently close to $\lambda_{1}$, we obtain a complete bifurcation diagram of (1.1), which is very interesting in a PDE context. We prove that when $0<a-\lambda_{1}<\delta$ for some $\delta>0$, (1.1) has exactly two positive steady state solutions $u_{1}$ and $u_{2}$ when $c \in\left(0, c_{2}\right)$, exactly one when $c=c_{2}$, and no nonnegative steady state solution when $c>c_{2}$ (see Fig. 2).

It is also interesting to compare the PDE model (1.6) and (1.3) to the ODE model with constant yield harvesting (see $[\mathrm{Cl}],[\mathrm{BC}]$ ):

$$
u^{\prime}=a u-b u^{2}-c, \quad u(0)=u_{0} .
$$

For (1.8), a complete bifurcation diagram in $(c, u)$ space can be drawn (see Fig. $3)$. Fix $a, b>0$; then there exists a critical number $c_{0}=a^{2} / 4 b$ such that when $0<c<c_{0}$, there are two equilibrium points

$$
u_{ \pm}=\frac{a \pm \sqrt{a^{2}-4 b c}}{2 b}
$$

such that $u(t) \rightarrow u_{+}$as $t \rightarrow \infty$ for any $u_{0}>u_{-}$and $u(t)<0$ when $t>T$ for any $u_{0}<u_{-}$; when $c>c_{0}$, for any $u_{0}>0$ we have $u(t)<0$ when $t>T$.

The main mathematical tools in the paper include comparison methods for semilinear elliptic equations and bifurcation theory in Banach spaces. The nonlinearity $f(x, u)=a u-b u^{2}-\operatorname{ch}(x)$ satisfies $f(x, 0)<0$ for $x \in \Omega$, which is often referred to as semi-positone nonlinearity, as the maximum principle does not hold in general. See CMS for a general survey for semi-positone problems.

When the harvesting term is homogeneous on $x$, there are more results available previously. When $p(t, x, u)=c$, a constant, see for example ACS, CS, OS] and $[\underline{S}$. We should mention that $h(x)=0$ on the boundary is not needed in the bifurcation type of results; it is only needed when we establish the existence and uniqueness of a solution such that $a u-b u^{2}>\operatorname{ch}(x)$, and so many of the results in this paper can also be proved for the case $h(x)>0$ for $x \in \bar{\Omega}$, in particular the case $p(t, x, u)=c$. Other types of predation terms have also been studied in the literature. Korman and Shi $[\mathrm{KS}$ studied the bifurcation diagram of steady state solutions of (1.4) and (1.3) with $p(t, x, u)=c u /(1+u)$ and $\Omega$ being the unit ball of 
dimension $1 \leq n \leq 4$, and a complete classification of precise bifurcation diagrams was achieved for all $a, c>0$. This type of nonlinearity is called Holling type II functional response of predator (see [Ho]). Other studies on the diffusive logistic equation can be found in [AB], [CC1], [CC2].

For a nonlinear operator $F$, we use $F_{u}$ as the partial derivative of $F$ with respect to the argument $u$. For a linear operator $L$, we use $N(L)$ as the null space of $L$ and $R(L)$ as the range of $L$. We introduce the anti-maximum principle and bifurcation theory in Sections 2.1 and 2.2. In Section 2.3, we recall results on the logistic equation, and we prove some a priori estimates in Section 2.4. In Section 3 , we prove the existence of solutions by comparison and bifurcation methods for all $a>\lambda_{1}$. The global bifurcation diagram for $0<a-\lambda_{1}<\delta$ is shown in Section 4.

\section{Preliminaries}

2.1. Maximum principle and anti-maximum principle. This section is a recollection of some preliminaries and results from previous works. First we recall the anti-maximum principle of Clément and Peletier [CP]. Let $\Omega \subset \mathbf{R}^{n}$ be a bounded smooth domain $\left(\partial \Omega\right.$ is of class $C^{2}$ ). Let $L$ denote the differential operator

$$
L u=-\sum_{i, j=1}^{n} a_{i j} \frac{\partial^{2} u}{\partial x_{i} \partial x_{j}}+\sum_{i=1}^{n} a_{i} \frac{\partial u}{\partial x_{i}}+a u,
$$

where $a_{i j} \in C(\bar{\Omega}), a_{i j}=a_{j i}$, and $\sum_{i, j=1}^{n} a_{i j}(x) \xi^{i} \xi^{j}>0$ for $x \in \bar{\Omega}$ and $\xi=\left(\xi^{i}\right) \in$ $\mathbf{R}^{n} \backslash\{0\}$, and $a_{i}, a \in L^{\infty}(\Omega)$.

Let $p>n$, let $X=\left\{u \in W^{2, p}(\Omega): u=0\right.$ on $\left.\partial \Omega\right\}$, and let $Y=L^{p}(\Omega)$. Let the operator $A: X \rightarrow Y$ be defined by $A u=L u$. Then from [CP], pages 220221 , we know that $A$ has a unique principal eigenvalue $\lambda_{1}(A)$, which is simple, and $A u=\lambda_{1}(A) u$ has a strict positive eigenfunction $\varphi_{1}$ such that

$$
\varphi_{1}(x)>0, \quad x \in \Omega, \frac{\partial \varphi_{1}}{\partial n}(x)<0, x \in \partial \Omega .
$$

Theorem $2.1([\overline{\mathrm{CP}}])$. Let $A$ be the elliptic operator defined above and let $\lambda_{1}(A)$ be its principal eigenvalue. Suppose that $f \in L^{p}(\Omega), p>n$, is such that $f>0$, and suppose $u$ satisfies the equation

$$
A u-\lambda u=f \quad \text { in } L^{p}(\Omega) .
$$

Then there exists $\delta_{f}>0$, which depends on $f$, such that if $\lambda_{1}(A)<\lambda<\lambda_{1}(A)+\delta_{f}$, then

$$
u(x)<0, \quad x \in \Omega, \quad \frac{\partial u}{\partial n}(x)>0, \quad x \in \partial \Omega,
$$

and if $\lambda<\lambda_{1}(A)$, then

$$
u(x)>0, \quad x \in \Omega, \quad \frac{\partial u}{\partial n}(x)<0, \quad x \in \partial \Omega .
$$

Here the result for $\lambda_{1}(A)<\lambda<\lambda_{1}(A)+\delta_{f}$ is called an anti-maximum principle, and the result for $\lambda<\lambda_{1}(A)$ is an extended maximum principle. 
2.2. Bifurcation theory. We use bifurcation theory to study the solution set, and our main tools are the implicit function theorem (see for example [CR1]) and two bifurcation theorems by Crandall and Rabinowitz [CR1], CR2], which we recall below. In all three theorems, $X$ and $Y$ are Banach spaces.

Theorem 2.2 (Implicit function theorem, CR1]). Let $\left(\lambda_{0}, u_{0}\right) \in \mathbf{R} \times X$ and let $F$ be a continuously differentiable mapping of an open neighborhood $V$ of $\left(\lambda_{0}, u_{0}\right)$ into $Y$. Let $F\left(\lambda_{0}, u_{0}\right)=0$. Suppose that $F_{u}\left(\lambda_{0}, u_{0}\right)$ is a linear homeomorphism of $X$ onto $Y$. Then the solutions of $F(\lambda, u)=0$ near $\left(\lambda_{0}, u_{0}\right)$ form a curve $\left(\lambda, u_{0}+\lambda w_{0}+\right.$ $z(\lambda)), \lambda \in\left(\lambda_{0}-\varepsilon, \lambda_{0}+\varepsilon\right)$ for some $\varepsilon>0$, where $w_{0}=-\left[F_{u}\left(\lambda_{0}, u_{0}\right)\right]^{-1}\left(F_{\lambda}\left(\lambda_{0}, u_{0}\right)\right)$ and $\lambda \mapsto z(\lambda) \in X$ is a continuously differentiable function near $\lambda=\lambda_{0}$ with $z\left(\lambda_{0}\right)=z^{\prime}\left(\lambda_{0}\right)=0$.

Theorem 2.3 (Bifurcation from a simple eigenvalue, CR1]). Let $\lambda_{0} \in \mathbf{R}$ and let $F$ be a continuously differentiable mapping of an open neighborhood $V \subset \mathbf{R} \times X$ of $\left(\lambda_{0}, 0\right)$ into $Y$. Suppose that

1. $F(\lambda, 0)=0$ for $\lambda \in \mathbf{R}$,

2. the partial derivative $F_{\lambda u}$ exists and is continuous,

3. $\operatorname{dim} N\left(F_{u}\left(\lambda_{0}, 0\right)\right)=\operatorname{codim} R\left(F_{u}\left(\lambda_{0}, 0\right)\right)=1$, and

4. $F_{\lambda u}\left(\lambda_{0}, 0\right) w_{0} \notin R\left(F_{u}\left(\lambda_{0}, 0\right)\right)$, where $w_{0} \in X$ spans $N\left(F_{u}\left(\lambda_{0}, 0\right)\right)$.

Let $Z$ be any complement of $\operatorname{span}\left\{w_{0}\right\}$ in $X$. Then there exist an open interval $I=(-\epsilon, \epsilon)$ and $C^{1}$ functions $\lambda: I \rightarrow \mathbf{R}, \psi: I \rightarrow Z$, such that $\lambda(0)=\lambda_{0}, \psi(0)=0$, and, if $u(s)=s w_{0}+s \psi(s)$ for $s \in I$, then $F(\lambda(s), u(s))=0$. Moreover, $F^{-1}(\{0\})$ near $\left(\lambda_{0}, 0\right)$ consists precisely of the curves $u=0$ and $(\lambda(s), u(s)), s \in I$.

We recall from $[\mathrm{S}]$ that in Theorem 2.3 , if $F$ is $C^{2}$ in $u$, then we have

$$
\lambda^{\prime}(0)=-\frac{\left\langle l, F_{u u}\left(\lambda_{0}, 0\right)\left[w_{0}, w_{0}\right]\right\rangle}{2\left\langle l, F_{\lambda u}\left(\lambda_{0}, 0\right)\right\rangle},
$$

where $\langle\cdot, \cdot\rangle$ is the duality between $Y$ and $Y^{*}$, the dual space of $Y$, and $l \in Y^{*}$ satisfies $N(l)=R\left(F_{u}\left(\lambda_{0}, 0\right)\right)$.

Theorem 2.4 (Saddle-node bifurcation at a turning point, [CR2]). Let $\left(\lambda_{0}, u_{0}\right) \in$ $\mathbf{R} \times X$ and let $F$ be a continuously differentiable mapping of an open neighborhood $V$ of $\left(\lambda_{0}, u_{0}\right)$ into $Y$. Suppose that

1. $\operatorname{dim} N\left(F_{u}\left(\lambda_{0}, u_{0}\right)\right)=\operatorname{codim} R\left(F_{u}\left(\lambda_{0}, u_{0}\right)\right)=1, N\left(F_{u}\left(\lambda_{0}, u_{0}\right)\right)=\operatorname{span}\left\{w_{0}\right\}$, and

2. $F_{\lambda}\left(\lambda_{0}, u_{0}\right) \notin R\left(F_{u}\left(\lambda_{0}, u_{0}\right)\right)$.

If $Z$ is a complement of $\operatorname{span}\left\{w_{0}\right\}$ in $X$, then the solutions of $F(\lambda, u)=F\left(\lambda_{0}, u_{0}\right)$ near $\left(\lambda_{0}, u_{0}\right)$ form a curve $(\lambda(s), u(s))=\left(\lambda_{0}+\tau(s), u_{0}+s w_{0}+z(s)\right)$, where $s \rightarrow$ $(\tau(s), z(s)) \in \mathbf{R} \times Z$ is a continuously differentiable function near $s=0$ and $\tau(0)=$ $\tau^{\prime}(0)=0, z(0)=z^{\prime}(0)=0$. Moreover, if $F$ is $k$ times continuously differentiable, so are $\tau(s)$ and $z(s)$.

We recall from [S] that in Theorem 2.4, if $F$ is $C^{2}$ in $u$, then we have

$$
\tau^{\prime \prime}(0)=-\frac{\left\langle l, F_{u u}\left(\lambda_{0}, u_{0}\right)\left[w_{0}, w_{0}\right]\right\rangle}{\left\langle l, F_{\lambda}\left(\lambda_{0}, u_{0}\right)\right\rangle},
$$

where $l \in Y^{*}$ satisfies $N(l)=R\left(F_{u}\left(\lambda_{0}, u_{0}\right)\right)$. 


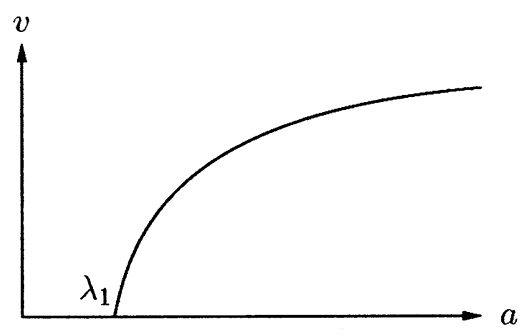

FIGURE 4.

2.3. Logistic equation. Here we recall the bifurcation diagram of the diffusive logistic equation. Indeed, we prove a more general result. Consider

$$
\begin{cases}\Delta u+a u-f(u)=0, & x \in \Omega, \\ u=0, & x \in \partial \Omega .\end{cases}
$$

Theorem 2.5. Assume that $f(u)$ satisfies

$$
\frac{d}{d u}\left(\frac{f(u)}{u}\right)>0, \text { for } u>0, \quad \lim _{u \rightarrow \infty} \frac{f(u)}{u}=\infty,
$$

and $f(0)=f^{\prime}(0)=0$. Then (2.8) has no positive solution if $a \leq \lambda_{1}$, and has exactly one positive solution $v_{a}$ if $a>\lambda_{1}$. Moreover, all $v_{a}$ 's lie on a smooth curve, $v_{a}$ is stable, and $v_{a}$ is increasing with respect to a.

Theorem 2.5 is more or less known to the experts in the field of semilinear elliptic equations, but we have not been able to find an exact reference; so here we provide a proof based on the implicit function theorem (Theorem 2.2) and bifurcation from a simple eigenvalue (Theorem 2.3. The key to proving Theorem 2.5] is the following lemma, which we will also use in this paper (this lemma was first proved in $\mathrm{ABC}$, and the form here was first proved in Shi and Yao $[\underline{S Y}]$ ).

Lemma 2.6. Suppose that $f: \Omega \times \mathbf{R}^{+} \rightarrow \mathbf{R}$ is a continuous function such that $f(x, s) / s$ is strictly decreasing for $s>0$ at each $x \in \Omega$. Let $w, v \in C(\bar{\Omega}) \cap C^{2}(\Omega)$ satisfy

(a) $\Delta w+f(x, w) \leq 0 \leq \Delta v+f(x, v)$ on $\Omega$,

(b) $w, v>0$ on $\Omega$ and $w \geq v$ on $\partial \Omega$,

(c) $\Delta v \in L^{1}(\Omega)$.

Then $w \geq v$ in $\bar{\Omega}$.

The stability of the solution is also an important subject in our study. We call a solution $u$ of

$$
\begin{cases}\Delta u+g(x, u)=0, & x \in \Omega, \\ u=0, & x \in \partial \Omega,\end{cases}
$$

a stable solution if all eigenvalues of

$$
\begin{cases}\Delta \psi+g_{u}(x, u) \psi=-\mu \psi, & x \in \Omega, \\ \psi=0, & x \in \partial \Omega,\end{cases}
$$

are strictly positive, which can be inferred if the principal eigenvalue $\mu_{1}(u)>0$. Otherwise $u$ is unstable. When $u$ is unstable, the number of negative eigenvalues 
$\mu_{i}$ of (2.11) is the Morse index $M(u)$ of $u$. If 0 is an eigenvalue of (2.11), then $u$ is a degenerate solution, otherwise nondegenerate.

Proof of Theorem [2.5. From (2.9) and $f(0)=f^{\prime}(0)=0$, we know that $f(u)>0$ for all $u>0$. Thus from (2.8) and (1.7), we have

$$
\int_{\Omega}\left(a-\lambda_{1}\right) u \phi_{1} d x=\int_{\Omega} f(u) \phi_{1} d x
$$

if $u$ is a positive solution of (2.8). So (2.8) has no positive solution if $a \leq \lambda_{1}$.

Next we apply Theorem 2.3 at $(a, u)=\left(\lambda_{1}, 0\right)$. Let $F(a, u)=\Delta u+a u-$ $b u^{2}$, where $a>0$ and $u \in X \equiv C^{2, \alpha}(\bar{\Omega})$, and let $Y=C^{\alpha}(\bar{\Omega}) . \quad(a, u)=(a, 0)$ is a line of trivial solutions of (2.8); at $\left(\lambda_{1}, 0\right), N\left(F_{u}\left(\lambda_{1}, 0\right)\right)=\operatorname{span}\left\{\phi_{1}\right\}$, and $R\left(F_{u}\left(\lambda_{1}, 0\right)\right)=\left\{\psi \in Y: \int_{\Omega} \psi \phi_{1} d x=0\right\}$, which is codimension $1 ; F_{a u}\left(\lambda_{1}, 0\right) \phi_{1}=$ $\lambda_{1} \phi_{1} \notin R\left(F_{u}\left(\lambda_{1}, 0\right)\right)$ since $\lambda_{1} \int_{\Omega} \phi_{1}^{2} d x \neq 0$. Thus by Theorem 2.3, near $\left(\lambda_{1}, 0\right)$, the solutions of (2.8) are on two branches $\Sigma_{0}=\{(a, 0)\}$ and $\Sigma_{1}=\{(a(s), v(s)):|s| \leq$ $\delta$, where $a(0)=\lambda_{1}, v(s)=s \phi_{1}+o\left(s^{2}\right)$; moreover $a(s)>\lambda_{1}$ for $s \in(0, \delta)$ from the last paragraph. Therefore there exists $\varepsilon>0$ such that for $a \in\left(\lambda_{1}, \lambda_{1}+\varepsilon\right)$, (2.8) has a positive solution $v_{a}$. We prove that any positive solution $(a, v)$ of (2.8) is stable. Let $\left(\mu_{1}(v), \psi_{1}\right)$ be the principal eigenpair of 2.11 for $g(x, v)=a v-f(v)$. Then from (2.11) and (2.8), we obtain

$$
-\mu_{1}(v) \int_{\Omega} \psi_{1} v d x=-\int_{\Omega}\left[f^{\prime}(v) v-f(v)\right] \psi_{1} d x
$$

Because of (2.9), $f^{\prime}(v) v-f(v)>0$ for $v>0$. Thus $\mu_{1}(v)>0$. In particular, any positive solution $(a, v)$ is nondegenerate. Therefore, at any positive solution $\left(a^{*}, v^{*}\right)$, we can apply Theorem 2.2 to $F(a, v)=0$, and all the solutions of $F(a, v)=0$ near $\left(a^{*}, v^{*}\right)$ are on a curve $(a, v(a))$ with $\left|a-a^{*}\right| \leq \varepsilon$ for some small $\varepsilon>0$. Hence the portion of $\Sigma_{1}$ with $s>0$ can be extended to a maximal set

$$
\Sigma_{1}=\left\{\left(a, v_{a}\right): a \in\left(\lambda_{1}, a_{M}\right)\right\}
$$

where $a_{M}$ is the supremum of all $a>a_{0}$ such that $v_{a}$ exists. We claim that $\lambda_{M}=\infty$. Suppose not. Then $\lambda_{M}<\infty$, and there are two possibilities: (a) $\lim _{a \rightarrow a_{M}^{-}}\left\|v_{a}\right\|_{X}=\infty$, or (b) $\lim _{a \rightarrow a_{M}^{-}} v_{a}=0$; otherwise we can extend $\Sigma_{1}$ further beyond $a_{M}$. The case (a) is impossible since $\lim _{u \rightarrow \infty} \frac{f(u)}{u}=\infty$; then, by the maximum principle,

$$
\|u\|_{\infty} \leq K, \quad \text { where } K=\max \left\{u>0: a>\frac{f(u)}{u}\right\} \text {. }
$$

The case (b) is not possible either, since if so, $a=a_{M}$ must be a point where a bifurcation from the trivial solutions $v=0$ occurs, $a_{M}$ must be an eigenvalue $\lambda_{i}$ of (1.7) with $i \geq 2$, and the eigenfunction $\phi_{i}$ is not of one sign, but the positive solution $v_{a}$ satisfies $v_{a} /\left\|v_{a}\right\|_{\infty} \rightarrow \phi_{i}$ as $a \rightarrow a_{M}^{-}$, which is a contradiction. Thus $a_{M}=\infty$.

We prove $v_{a}$ is increasing with respect to $a$. Since $v_{a}$ is differentiable with respect to $a$ (as a consequence of the implicit function theorem), then $\frac{d v_{a}}{d a}$ satisfies

$$
\Delta \frac{d v_{a}}{d a}-a \frac{d v_{a}}{d a}+f^{\prime}\left(v_{a}\right) \frac{d v_{a}}{d a}=-v_{a} \leq 0
$$


and $v_{a}$ is stable; so $\mu_{1}\left(v_{a}\right)>0$. Then, by Theorem 2.1, $\frac{d v_{a}}{d a} \geq 0$. Finally, by Lemma 2.6, (2.8) has at most one positive solution for any possible $\lambda>0$, which completes the proof.

2.4. Some a priori estimates. We close this section with some results on the dependence of solutions on the parameter $a>0$. First we prove some nonexistence results:

Proposition 2.7. 1. If $a \leq \lambda_{1}$ and $c \geq 0$, (1.1) has no nonnegative solution except $u=0$ when $c=0$.

2. If $a>\lambda_{1}$ and

$$
c>\frac{a\left(a-\lambda_{1}\right) \int_{\Omega} \phi_{1} d x}{b \int_{\Omega} h \phi_{1} d x},
$$

then (1.1) has no nonnegative solution.

Proof. (1) Multiplying (1.1) by $\phi_{1}$, and integrating over $\Omega$, we obtain

$$
\left(a-\lambda_{1}\right) \int_{\Omega} u \phi_{1} d x=b \int_{\Omega} u^{2} \phi_{1} d x+c \int_{\Omega} h(x) \phi_{1} d x .
$$

Since $u \geq 0, \phi_{1}>0, b, c \geq 0$ and $a-\lambda_{1} \leq 0$, then the equality can only be achieved when $u \equiv 0$ and $c=0$.

(2) From the maximum principle, we have $\|u\|_{\infty} \leq a / b$ for any nonnegative solution $u$. Hence from (2.15), we obtain

$$
c \int_{\Omega} h(x) \phi_{1} d x \leq\left(a-\lambda_{1}\right) \int_{\Omega} u \phi_{1} d x \leq \frac{a\left(a-\lambda_{1}\right)}{b} \int_{\Omega} \phi_{1} d x,
$$

a contradiction when (2.14) holds.

So $a>\lambda_{1}$ is a necessary condition for the existence of nonnegative solutions. When $a>\lambda_{1}$, we have the following estimate:

Proposition 2.8. If $a>\lambda_{1}, c \geq 0$, and $u$ is a nonnegative solution to (1.1), then

$$
\|u\|_{W^{2,2}(\Omega)}^{2} \leq C_{1}\left(a-\lambda_{1}\right)^{2},
$$

where $C_{1}$ is a positive constant depending only on $\Omega, a, b$ and $h$.

Proof. Multiplying (1.1) by $u$, and integrating over $\Omega$, we obtain

$$
-\int_{\Omega}|\nabla u|^{2} d x+a \int_{\Omega} u^{2} d x=b \int_{\Omega} u^{3} d x+c \int_{\Omega} h(x) u d x>0 .
$$

Thus

$$
\|u\|_{W^{1,2}(\Omega)}^{2} \leq(a+1) \int_{\Omega} u^{2} d x .
$$

On the other hand, from (2.18), we obtain

$$
\left(a-\lambda_{1}\right) \int_{\Omega} u^{2} d x \geq-\int_{\Omega}|\nabla u|^{2} d x+a \int_{\Omega} u^{2} d x>b \int_{\Omega} u^{3} d x
$$

here in the first inequality, we use the fact that $\int_{\Omega}|\nabla u|^{2} d x \geq \lambda_{1} \int_{\Omega} u^{2} d x$. Then, using Schwarz inequality inductively and (2.20), we can obtain

$$
\int_{\Omega} u^{n} d x \leq\left(\frac{a-\lambda_{1}}{b}\right)^{n}|\Omega|,
$$


for $n=1,2$ and 3, which, together with (2.19), implies

$$
\|u\|_{W^{1,2}(\Omega)}^{2} \leq(a+1)\left(\frac{a-\lambda_{1}}{b}\right)^{2}|\Omega| .
$$

Finally, from Lemma 2.6, we have $b / a \geq u(x)$ and $a u(x)-b u^{2}(x) \geq 0$ for all $x \in \Omega$; thus $|\Delta u(x)| \leq\left|a u(x)-b u^{2}(x)\right|+|\operatorname{ch}(x)| \leq|a u(x)|+|\operatorname{ch}(x)|$ for any $x \in \Omega$. Then by $\|h\|_{\infty}=1$ and (2.14), we have

$$
\begin{aligned}
& \|\Delta u\|_{L^{2}(\Omega)}^{2} \leq a^{2}|| u \|_{L^{2}(\Omega)}^{2}+c^{2}|\Omega| \\
& \quad \leq a^{2}(a+1)\left(\frac{a-\lambda_{1}}{b}\right)^{2}|\Omega|+\left[\frac{a\left(a-\lambda_{1}\right) \int_{\Omega} \phi_{1} d x}{b \int_{\Omega} h \phi_{1} d x}\right]^{2}|\Omega| \\
& \quad \leq C_{2}\left(a-\lambda_{1}\right)^{2},
\end{aligned}
$$

where $C_{2}>0$ depends on $a, b, h$ and $\Omega$, and, from standard elliptic estimates,

$$
\|u\|_{W^{2,2}(\Omega)}^{2} \leq C_{3}\left(\|u\|_{W^{1,2}(\Omega)}^{2}+\|\Delta u\|_{L^{2}(\Omega)}^{2}\right) \leq C_{1}\left(a-\lambda_{1}\right)^{2},
$$

where $C_{3}>0$ depends only on $\Omega$.

\section{Existence of LARGE AND SMALl SOlutions}

To consider the problem in an abstract setting, we define

$$
F(c, u)=\Delta u+a u-b u^{2}-\operatorname{ch}(x),
$$

where $c \in \mathbf{R}, u \in W^{2,2}(\Omega) \cap W_{0}^{1,2}(\Omega)$. Clearly, (1.1) has a solution $(c, u)$ if and only if $F(c, u)=0$. We remark that, though we consider the equation in Sobolev space $W^{2,2}(\Omega)$, all solutions to the equation (1.1) are classical solutions belonging to $C^{2, \alpha}(\bar{\Omega})$, since $g(x, u)=a u-b u^{2}-c h(x)$ belongs to $C^{\alpha}(\bar{\Omega})$ by our assumption. In particular, all solutions and related functions involved in the proofs also belong to $W^{2, p}(\Omega)$ for $p>n$, which is the necessary condition for applying the anti-maximum principle.

Our main results in this section are the following:

Theorem 3.1. Suppose that $a>\lambda_{1}$ and $b>0$. Then there exists $c_{1}=c_{1}(a, b)$ such that for $0<c<c_{1}$, (1.1) has a positive solution $u_{1}(x, c)$ such that

$$
a u_{1}(x, c)-b u_{1}^{2}(x, c)>\operatorname{ch}(x)>0 .
$$

Theorem 3.2. Suppose that $a>\lambda_{1}$ and $b>0$. Then there exists $c_{2}(a, b)>c_{1}$ such that

1. for $0<c<c_{2}$, (1.1) has a maximal positive solution $u_{1}(x, c)$ such that for any solution $v(x, c)$ of (1.1), $u_{1} \geq v$;

2. for $c>c_{2}$, (1.1) has no positive solution;

3. for $0<c<c_{2}, u_{1}(\cdot, c)$ is stable with $\mu_{1}\left(u_{1}(\cdot, c)\right)>0$; and

4. $u_{1}(\cdot, c)$ is decreasing with respect to the parameter $c$ for $c \in\left(0, c_{2}\right)$.

Theorem 3.3. Suppose that $a>\lambda_{1}, a \neq \lambda_{i}$, and $b>0$. Then there exists $c_{3} \in$ $\left(0, c_{2}\right)$ such that

1. for $c \in\left(0, c_{3}\right)$, (1.1) has a second solution $u_{2}(x, c) \neq u_{1}(x, c)$ such that $\lim _{c \rightarrow 0^{+}}\left\|u_{2}(\cdot, c)\right\|_{W^{2,2}(\Omega)}=0$, and

2. if in addition $a \in\left(\lambda_{1}, \lambda_{1}+\delta_{h}\right)$ for some $\delta_{h}>0$, then $u_{2}(x, c)>0$ for $x \in \Omega$. 
Theorem 3.4. Suppose that $a>2 \lambda_{1}$ and $b>0$. Then there exists $0<c_{4}<c_{1}$ such that for $c \in\left(0, c_{4}\right)$, (1.1) has a unique positive solution (which must be $u_{1}(x, c)$ ) satisfying

$$
\lambda_{1} u_{1}(x, c) \geq \operatorname{ch}(x), \quad x \in \Omega .
$$

Proof of Theorem 3.1. We use the method of sup-sub solutions. Let $z_{\lambda}$ be the unique solution of

$$
\begin{cases}\Delta z_{\lambda}+\lambda z_{\lambda}=1, & x \in \Omega, \\ z_{\lambda}=0, & x \in \partial \Omega,\end{cases}
$$

where $\lambda \in\left(\lambda_{1}, \lambda_{1}+\delta_{1}\right)$, and $\delta_{1}=\delta_{1}(\Omega)>0$ is the constant in Theorem 2.1 for the validity of the anti-maximum principle. Then from Theorem 2.1. we have

$$
z_{\lambda}(x)>0, \quad x \in \Omega, \frac{\partial z_{\lambda}}{\partial n}(x)<0, x \in \partial \Omega .
$$

We construct a subsolution $\Psi(x)$ of (1.1) using $z_{\lambda}$ such that

$$
\lambda_{1} \Psi(x) \geq \operatorname{ch}(x) .
$$

Fix $\lambda_{*} \in\left(\lambda_{1}, \min \left\{a, \lambda_{1}+\delta_{1}\right\}\right)$. Let

$$
\begin{aligned}
& \alpha=\left\|z_{\lambda_{*}}\right\|_{\infty}, \quad K_{0}=\inf \left\{K: \lambda_{1} K z_{\lambda_{*}}(x) \geq h(x)\right\}, \\
& K_{1}=\max \left\{1, K_{0}\right\} .
\end{aligned}
$$

Note that $K_{0}>0$ exists from (3.5). Define $\Psi(x)=K c z_{\lambda_{*}}(x)$, where $K>0$ is to be determined later. We will choose $K>0$ and $c>0$ properly so that $\Psi$ is a subsolution. First we require that $K \geq K_{1}$; then $\lambda_{1} \Psi(x) \geq \operatorname{ch}(x)$. We have

$$
\begin{aligned}
\Delta \Psi & +a \Psi-b \Psi^{2}-\operatorname{ch}(x) \\
& =-K c\left(\lambda_{*} z_{\lambda_{*}}-1\right)+a K c z_{\lambda_{*}}-b\left(K c z_{\lambda_{*}}\right)^{2}-c h(x) \\
& \geq-K c\left(\lambda_{*} z_{\lambda_{*}}-1\right)+a K c z_{\lambda_{*}}-b\left(K c z_{\lambda_{*}}\right)^{2}-c \\
& =c\left[-b c\left(K z_{\lambda_{*}}\right)^{2}+\left(a-\lambda_{*}\right)\left(K z_{\lambda_{*}}\right)+(K-1)\right] .
\end{aligned}
$$

Define $H(x)=-b c x^{2}+\left(a-\lambda_{*}\right) x+(K-1)$. Thus $\Psi(x)$ is a subsolution if $H(x) \geq 0$ for all $x \in[0, K \alpha]$. Notice that $H(0)=K-1>0, H^{\prime}(0)=a-\lambda_{*} \geq 0$, and $H^{\prime \prime}(0)=-2 b c<0$. Hence $H(x) \geq 0$ for all $x \in[0, K \alpha]$ if $H(K \alpha) \geq 0$, which is equivalent to

$$
\left(a-\lambda_{*}\right) K \alpha+(K-1) \geq b c(K \alpha)^{2},
$$

or

$$
c \leq \frac{\left(a-\lambda_{*}\right) K \alpha+(K-1)}{b(K \alpha)^{2}} .
$$

We define

$$
c_{1} \equiv c_{1}(a, b)=\sup _{y \geq K_{1}} \frac{\left(a-\lambda_{*}\right) y \alpha+(y-1)}{b \alpha^{2} y^{2}}>0 .
$$

Then when $c \in\left(0, c_{1}\right)$, there exists $\widetilde{K} \geq K_{1}$ such that

$$
c \leq \frac{\left(a-\lambda_{*}\right) \widetilde{K} \alpha+(\widetilde{K}-1)}{b \alpha^{2} \widetilde{K}^{2}},
$$

and hence $\Psi(x)=\widetilde{K} c z_{\lambda_{*}}$ turns out to be a subsolution. On the other hand, it is easy to see that any large positive constant $C$ is a supersolution to (1.1) for fixed 
$a, b, c>0$. Therefore, from the standard result of the sub-sup solution method (see for example [Sa] $)$, when $c \in\left(0, c_{1}\right)$, there exists a solution $u_{1}(\cdot, c)$ of (1.1) satisfying $C \geq u_{1}(x, c) \geq \Psi(x) \geq\left(c / \lambda_{1}\right) h(x)$. Since $a>\lambda_{1}$, thus $a u_{1}(x, c)>\lambda_{1} u_{1}(x, c) \geq$ $\lambda_{1} \Psi(x) \geq \operatorname{ch}(x)$.

Finally we prove that if we choose $c_{1}$ smaller, then

$$
a u_{1}(x, c)-b u_{1}^{2}(x, c)>\operatorname{ch}(x) .
$$

Indeed, from a simple calculation, we can see that (3.13) will be satisfied if

$$
u_{1}(x, c)>\frac{a-\sqrt{a^{2}-4 b c h(x)}}{2 b}
$$

and

$$
u_{1}(x, c)<\frac{a+\sqrt{a^{2}-4 b c h(x)}}{2 b} .
$$

To prove (3.14), we notice that from our construction of $u_{1}, u_{1}(x, c) \geq \operatorname{ch}(x) / \lambda_{1}$. Hence (3.14) will be satisfied if

$$
\frac{\operatorname{ch}(x)}{\lambda_{1}}>\frac{a-\sqrt{a^{2}-4 b c h(x)}}{2 b},
$$

which is true if

$$
\lambda_{1} a-\lambda_{1}^{2}>b \operatorname{ch}(x)
$$

Therefore if we require

$$
c<\frac{\lambda_{1} a-\lambda_{1}^{2}}{b\|h\|_{\infty}}
$$

then (3.14) holds. To prove (3.15), we consider the equation (1.1) with $c=0$ :

$$
\begin{cases}\Delta u+a u-b u^{2}=0, & x \in \Omega, \\ u=0, & x \in \partial \Omega .\end{cases}
$$

From Theorem 2.5 we know that (3.17) has a unique positive solution $v_{a}$ when $a>\lambda_{1}$. Let $u$ be any nonnegative solution of (1.1). Then

$$
\Delta v_{a}+a v_{a}-b v_{a}^{2}=0<\operatorname{ch}(x)=\Delta u_{1}+a u_{1}-b u_{1}^{2}
$$

and $u_{1}=v_{a}=0$ on the boundary. By Lemma 2.6, $v_{a}(x) \geq u_{1}(x)$ for $x \in \Omega$, since $g(u)=a u-b u^{2}$ satisfies $(g(u) / u)^{\prime}<0$ for $u \geq 0$. So (3.15) can be achieved if

$$
v_{a}(x)<\frac{a+\sqrt{a^{2}-4 b c h(x)}}{2 b} .
$$

From a simple calculation, we can see that $(3.18)$ is true if

$$
c \leq \frac{a^{2}-\left(2 b \mid\left\|v_{a}\right\|_{\infty}-a\right)^{2}}{4 b|| h \|_{\infty}} .
$$

Therefore, we choose $c_{1}$ such that both (3.16) and (3.19) are satisfied. Then (3.13) holds.

Proof of Theorem 3.2. We follow a similar proof in Shi and Shivaji [SS], as well as the earlier work by Shi and Yao $[\mathrm{SY}$.

From the last part of the proof of Theorem 3.1, whenever (1.1) has a nonnegative solution $u$, then for the same parameters $(a, c)$, (1.1) also has a maximal solution $u_{1}(\cdot, c)$, which can be constructed as follows. We take $v_{a}$ as a supersolution, any 
solution $u$ as a subsolution, and make the iteration sequences as in the sub-sup solution method. Then we obtain a solution $u_{1}$ in between $v_{a}$ and $u$; in particular, $u_{1} \geq u$. Since $u$ can be any solution, then the limit of the iterated sequence starting from $v_{a}$ is the maximal solution. Clearly such $u_{1}$ is uniquely determined. (See details in [SY] or [SS].)

Thus we obtain a maximal positive solution $u_{1}(x, c)$ for $c \in\left(0, c_{1}\right)$, where $c_{1}$ is defined in Theorem 3.1 since we have proved (1.1) has a solution when $c \in\left(0, c_{1}\right)$ in Theorem 3.1. Moreover, it is clear that if $a>\lambda_{1}$ is fixed, then

$$
\lim _{c \rightarrow 0^{+}}\left\|u_{1}(x, c)-v_{a}(x)\right\|_{C^{2, \alpha}(\bar{\Omega})}=0 .
$$

Thus $\left(c, u_{1}(\cdot, c)\right)$ is coincident with the branch of solutions of (1.1) perturbed from $v_{a}$ by the implicit function theorem (Theorem 2.2). We define

$$
c_{2}=\sup \{c>0: \text { (1.1) has a nonnegative solution with this } c\} .
$$

Then $c_{2}<\infty$ from Proposition 2.7. Then for $c \in\left(0, c_{2}\right)$, (1.1) has a maximal positive solution $u_{1}(x, c)$, and $u_{1}(\cdot, c)$ is continuous with respect to $c$; from the construction of $u_{1}(\cdot, c)$.

We prove that

$$
\mu_{1}\left(u_{1}(\cdot, c)\right)>0, \quad \frac{\partial u_{1}(x, c)}{\partial c}<0, \quad x \in \Omega .
$$

First, (3.21) holds for $c=0$. From Theorem[2.5, we have $\mu_{1}\left(u_{1}(\cdot, 0)\right)=\mu_{1}\left(v_{a}(\cdot)\right)>$ 0 . From Theorem [2.2, $\partial u_{1}(x, 0) / \partial c=-\left[F_{u}\left(0, v_{a}\right)\right]^{-1}\left(F_{c}\left(0, v_{a}\right)\right)$ is the solution of

$$
\Delta w+a w-2 b v_{a} w=h(x), \quad x \in \Omega, \quad w=0, \quad x \in \partial \Omega .
$$

Since $\mu_{1}\left(v_{a}(\cdot)\right)>0$, then from Theorem 2.1,

$$
\frac{\partial u_{1}(x, 0)}{\partial c}<0, \quad x \in \Omega, \quad \frac{\partial}{\partial n} \frac{\partial u_{1}(x, 0)}{\partial c}>0, x \in \partial \Omega .
$$

Since $u_{1}(\cdot, c)$ is continuous with respect to $c$, then (3.21) holds when $c \in\left(0, c^{*}\right)$ for some $c^{*} \in\left(0, c_{2}\right)$. We claim that (3.21) holds for all $c \in\left(0, c_{2}\right)$. Suppose this is not true; then at some $c_{*} \in\left(0, c_{2}\right)$, one of the statements in (3.21) is not true. If we have $\mu_{1}\left(u_{1}\left(\cdot, c_{*}\right)\right)>0$, then using the same proof as above, we can show that $\partial u_{1}\left(x, c_{*}\right) / \partial c<0$. Thus $\mu_{1}\left(u_{1}\left(\cdot, c_{*}\right)\right)=0$. We apply Theorem 2.4 at $\left(c_{*}, u_{*}\right)$, where $u_{*}=u_{1}\left(\cdot, c_{*}\right)$. Since 0 is the principal eigenvalue of $F_{u}\left(c_{*}, u_{*}\right)$, then $\operatorname{dim} N\left(F_{u}\left(c_{*}, u_{*}\right)\right)=\operatorname{codim} R\left(F_{u}\left(c_{*}, u_{*}\right)\right)$, and $N\left(F_{u}\left(c_{*}, u_{*}\right)\right)=\operatorname{span}\left\{w_{0}\right\}$, where $w_{0}$ is a solution of

$$
\Delta w+a w-2 b u_{*} w=0, \quad x \in \Omega, \quad w=0, \quad x \in \partial \Omega .
$$

Also $F_{c}\left(c_{*}, u_{*}\right) \notin R\left(F_{u}\left(c_{*}, u_{*}\right)\right)$, since $F_{c}\left(c_{*}, u_{*}\right)=-h(x)$ and $-\int_{\Omega} h(x) w_{0}(x) d x \neq$ 0 , while $h>0$ and $w_{0}>0$. Therefore near $\left(c_{*}, u_{*}\right)$, the solutions of (1.1) form a curve $(c(s), u(s))=\left(c_{*}+o(|s|), u_{*}+s w_{0}+o(|s|)\right)$ with $|s|<\delta$. Moreover, by (2.6),

$$
c^{\prime \prime}(0)=-\frac{2 b \int_{\Omega} w_{0}^{3}(x) d x}{\int_{\Omega} h(x) w_{0}(x) d x}<0 .
$$

Thus (1.1) has no solution near $\left(c_{*}, u_{*}\right)$ when $c \in\left(c_{*}, c_{*}+\delta_{1}\right)$ for some $\delta_{1}>0$. However, $c_{*}<c_{2}$, and $u_{1}(\cdot, c)$ is continuous with respect to $c$; so (1.1) has at least one solution $u_{1}(\cdot, c)$ for $c \in\left(c_{*}, c_{*}+\delta_{1}\right)$ which is also near $u_{*}$. That is a contradiction. Hence (3.21) holds for all $c \in\left(0, c_{2}\right)$. 
Proof of Theorem 3.3. We apply the implicit function theorem (Theorem 2.2). Let $F(c, u)$ be defined as in (3.1). At $(c, u)=(0,0)$, we have $F_{u}(0,0) w=\Delta w+a w$. For $a \neq \lambda_{i}, F_{u}(0,0)$ is an isomorphism from $X$ to $Y$. Fix $a \neq \lambda_{i}$; then the solution set of (1.I) near $(0,0)$ is of form $\left(c, u_{2}(\cdot, c)\right)$ for $c \in\left(-\delta_{1}, \delta_{1}\right), u_{2}(\cdot, 0)=0$, and $u_{2}(\cdot, c)=c w_{0}+o(|c|)$, where $w_{0}=-\left[F_{u}(0,0)\right]^{-1}\left(F_{c}(0,0)\right)$ is the solution of

$$
\Delta w+a w=h(x), \quad x \in \Omega, \quad w=0, \quad x \in \partial \Omega .
$$

Since $h(\cdot) \in C^{\alpha}(\bar{\Omega})$, then $h \in L^{p}(\Omega)$ for any $p>n$. Suppose that $\delta_{h}>0$ is the constant such that the anti-maximum principle holds for $A=-\Delta, f=-h<0$; then, from Theorem 2.1 $w_{0}(x)>0$ for $x \in \Omega$ and $\partial_{n} w_{0}(x)<0$ for $x \in \partial \Omega$. In particular, $u_{2}(\cdot, c)>0$ for $c \in\left(0, c_{3}\right)$.

Proof of Theorem 3.4. Suppose that $u$ is a nonnegative solution of (1.1) which satisfies (3.3). Then from (1.1) and $\Delta \phi_{1}+\lambda_{1} \phi_{1}=0$, we obtain

$$
\int_{\Omega}\left[a-\lambda_{1}-b u\right] u \phi_{1} d x-c \int_{\Omega} h \phi_{1} d x=0 .
$$

Let $a=2 \lambda_{1}+\delta$ for some $\delta>0$. Then, using (3.3) and (3.23), we obtain

$$
\int_{\Omega}(\delta-b u) u \phi_{1} d x<0
$$

In particular,

$$
\|u\|_{\infty}>\frac{\delta}{b}
$$

Since nonnegative solutions of (1.1) are bounded by Proposition 2.8, and when $c=0$ the only nonnegative solutions of (1.1) are 0 and $v_{a}$, then for $c>0$ sufficiently close to 0 , the only possible nonnegative solutions are perturbations of 0 or $v_{a}$. In that case, nonnegative solutions of (1.1) can only be $u_{1}(x, c)$ or $u_{2}(x, c)$. From the proof of Theorem 3.3, $u_{2}(x, c)=c w_{0}+o(|c|)$; thus if we choose $c>0$ also satisfying

$$
c<\frac{\delta}{2 b\left\|w_{0}\right\|_{\infty}},
$$

then $\left\|u_{2}(\cdot, c)\right\|_{\infty}<\delta / b$. In particular, $u_{2}$ does not satisfy (3.3), which implies the uniqueness of $u_{1}(x, c)$.

Remarks. 1. In Theorem 3.2 there is no information on the solution(s) when $c=c_{2}$. It is easy to show that

$$
u_{1}\left(x, c_{2}\right)=\lim _{c \rightarrow c_{2}^{-}} u_{1}(x, c)
$$

is a classical nonnegative solution of (1.1) for $c=c_{2}$, and $\left(c_{2}, u_{1}\left(x, c_{2}\right)\right)$ is a degenerate solution such that $\mu_{1}\left(u_{1}\left(\cdot, c_{2}\right)\right)=0$. However it is not clear if

$$
u_{1}\left(x, c_{2}\right)>0, x \in \Omega, \frac{\partial u_{1}\left(x, c_{2}\right)}{\partial n}<0, x \in \partial \Omega,
$$

is true. Note that Theorem 2.4 can be applied at $\left(c_{2}, u_{1}\left(x, c_{2}\right)\right)$ in a way similar to the argument in the proof of Theorem 3.2 even when (3.24) is not true. But then we do not know whether the solutions on the lower branch are positive or not. In Section 4, we show that (3.24) is true if $a$ is close enough to $\lambda_{1}$, and further study on this problem will be reported in [SS]. 
2. In Theorem 3.3, $u_{2}(\cdot, c)$ can still be positive even when $a$ is far away from $\lambda_{1}$. From the proof of Theorem 3.3 it is sufficient to show that $w_{0}>0$ for the solution $w_{0}$ of (3.22). Consider the following example: $n=1, \Omega=(0, \pi)$ and $h(x)=\sin x$. Then $w_{0}$ is the solution of

$$
w^{\prime \prime}+a w=\sin x, \quad x \in(0, \pi), \quad w(0)=w(\pi)=0 .
$$

It is easy to verify that $w_{0}(x)=\sin x /(a-1)$ for any $a>\lambda_{1}=1$. In that case, for any $a \neq \lambda_{i}=i^{2}, u_{2}(\cdot, c)$ is a positive solution for small $c>0$.

\section{Global bifurcation}

In this section, we show that when $a$ is slightly greater than $\lambda_{1}$, a more precise global bifurcation diagram of positive solutions to (1.1) can be obtained by using some ideas from Shi $[\mathrm{S}]$. In particular, we show that the branch of large solutions connects to the branch of small solutions (bifurcating from 0 as in Theorem 3.3), and the shape of the bifurcation diagram is exactly $\supset$-shaped as in the scalar ODE case. (See Fig. 2.)

Theorem 4.1. If $b>0$, then there exists $\delta_{2}>0$ such that for $a \in\left(\lambda_{1}, \lambda_{1}+\delta_{2}\right)$,

1. (1.1) has exactly two positive solutions $u_{1}(\cdot, c)$ and $u_{2}(\cdot, c)$ for $c \in\left[0, c_{2}\right)$, exactly one positive solution $u_{1}(\cdot, c)$ for $c=c_{2}$, and no positive solution for $c>c_{2}$;

2. the Morse index $M(u)$ is 1 for $u=u_{2}(\cdot, c), c \in\left[0, c_{2}\right)$, and $u_{1}\left(\cdot, c_{2}\right)$ is degenerate with $\mu_{1}\left(u_{1}\left(\cdot, c_{2}\right)\right)=0$;

3. all solutions lie on a smooth curve $\Sigma$ that, on $(c, u)$ space, starts from $(0,0)$, continues to the right, reaches the unique turning point at $c=c_{2}$ where it turns back, then continues to the left without any turnings until it reaches $\left(0, v_{a}\right)$, where $v_{a}$ is the unique positive solution of (1.1) with $c=0$.

To prove Theorem 4.1, we first prove the following lemmas:

Lemma 4.2. For $a \in\left(\lambda_{1}, \lambda_{1}+\delta_{3}\right)$, (1.1) has a unique degenerate solution, which is positive.

Proof. We apply the implicit function theorem in a different way here. Define

$$
F(a, c, u)=\Delta u+a u-b u^{2}-\operatorname{ch}(x),
$$

and

$$
H(a, c, u, w)=\left(\begin{array}{c}
F(a, c, u) \\
F_{u}(a, c, u)[w]
\end{array}\right)=\left(\begin{array}{c}
\Delta u+a u-b u^{2}-c h(x) \\
\Delta w+a w-2 b u w
\end{array}\right),
$$

where $a, c \in \mathbf{R}, b>0$ is fixed, $u \in X \equiv W^{2,2}(\Omega) \cap W_{0}^{1,2}(\Omega), w \in X_{1}=\{u \in$ $\left.X: \int_{\Omega} u^{2}(x) d x=1\right\}, Y=L^{2}(\Omega)$. Then (1.1) has a degenerate solution $(a, c, u)$ if and only if $H(a, c, u, w)=0$ has a nontrivial solution $(a, c, u, w)$. We consider the operator $H$ in a neighborhood $M$ of $\left(\lambda_{1}, 0,0, \phi_{1}\right)$ :

$$
\begin{aligned}
M= & \left\{(a, c, u, w) \in \mathbf{R}^{2} \times X \times X_{1}:\left|a-\lambda_{1}\right|<\delta_{4},|c| \leq \delta_{4},\right. \\
& \left.\|u\| \leq \delta_{4},\left\|w-\phi_{1}\right\| \leq \delta_{4}\right\},
\end{aligned}
$$

where $\delta_{4}$ is a positive constant and $\|\cdot\|$ is the norm of $W^{2,2}(\Omega)$. We prove that there exists $\delta_{5}>0$ such that $H(a, c, u, w)=0$ has a unique solution in $M$ for each 
$a \in\left(\lambda_{1}-\delta_{5}, \lambda_{1}+\delta_{5}\right)$. Let

$$
\begin{aligned}
& K[\tau, v, \psi]=H_{(c, u, w)}\left(\lambda_{1}, 0,0, \phi_{1}\right)[\tau, v, \psi] \\
= & \left(\begin{array}{c}
\tau F_{c}\left(\lambda_{1}, 0,0\right)+F_{u}\left(\lambda_{1}, 0,0\right)[v] \\
\tau F_{c u}\left(\lambda_{1}, 0,0\right)\left[w_{0}\right]+F_{u u}\left(\lambda_{1}, 0,0\right)\left[v, \phi_{1}\right]+F_{u}\left(\lambda_{1}, 0,0\right)[\psi]
\end{array}\right),
\end{aligned}
$$

where

$$
\begin{aligned}
& F_{u}(a, c, u)[v]=\Delta v+a v-2 b u v, \\
& F_{c}(a, c, u)=-h(x), \quad F_{c u}(a, c, u)[v]=0, \\
& F_{u u}(a, c, u)[v, \psi]=-2 b v \psi,
\end{aligned}
$$

$\tau \in \mathbf{R}, v \in X$ and $\psi \in X_{2} \equiv T_{\phi_{1}}\left(X_{1}\right)=\left\{u \in X: \int_{\Omega} u \phi_{1} d x=0\right\}$, the tangent space of $X_{1}$ at $\phi_{1}$. We prove that $K$ is a homeomorphism.

First we prove that $K$ is injective. Suppose there exists $(\tau, v, \psi)$ such that $K(\tau, v, \psi)=(0,0)$. Then $(\tau, v, \psi)$ satisfies

$$
\begin{aligned}
& \Delta v+\lambda_{1} v-\tau h(x)=0, \\
& \Delta \psi+\lambda_{1} \psi-2 b v \phi_{1}=0 .
\end{aligned}
$$

We multiply (4.8) by $\phi_{1}$, and integrate over $\Omega$, to get

$$
\tau \int_{\Omega} h(x) \phi_{1} d x=0
$$

Since $h>0$ and $\phi_{1}>0$, then $\tau=0$. Thus $v=k \phi_{1}$ for some $k \in \mathbf{R}$. We multiply (4.9) by $\phi_{1}$, and integrate over $\Omega$, to get

$$
2 b k \int_{\Omega} \phi_{1}^{3} d x=0 .
$$

Thus $k=0$ and $\Delta \psi+\lambda_{1} \psi=0$. But $\psi \in X_{2}$, so $\psi=0$. So $(\tau, v, \psi)=0$, and $K$ is injective.

Next we prove $K$ is surjective. Let $(f, g) \in Y \times Y$; then we need to find $(\tau, v, \psi) \in$ $\mathbf{R} \times X \times X_{2}$ such that

$$
\begin{aligned}
& \Delta v+\lambda_{1} v-\tau h(x)=f, \\
& \Delta \psi+\lambda_{1} \psi-2 b v \phi_{1}=g .
\end{aligned}
$$

Again we multiply (4.12) by $\phi_{1}$, and integrate over $\Omega$; then

$$
\tau=-\frac{\int_{\Omega} f \phi_{1} d x}{\int_{\Omega} h \phi_{1} d x}
$$

By the Fredholm alternative, (4.12) has a unique solution $v_{1}$ with $\tau$ given by (4.14) such that $\int_{\Omega} v_{1} \phi_{1} d x=0$. We substitute $v=v_{1}+k \phi_{1}$ in (4.13), multiply (4.13) by $\phi_{1}$, and integrate over $\Omega$; then $k$ is determined by

$$
-2 b \int_{\Omega} v_{1} \phi_{1}^{2} d x-2 b k \int_{\Omega} \phi_{1}^{3} d x=\int_{\Omega} g \phi_{1} d x
$$

Finally, $\psi \in X_{2}$ can be uniquely solved for in (4.13) once $k$ is determined as in (4.15). Therefore, $(f, g) \in R(K)$, and $K$ is a bijection.

On the other hand, since $F$ is twice differentiable, then $K$ is continuous, and $K^{-1}$ is also continuous by the open mapping theorem of Banach ( $\left.\mathrm{Y}, \mathrm{pg} .75\right)$. Thus $K$ is a linear homeomorphism, and by the implicit function theorem (Theorem[2.2), 
the solutions of $H(a, c, u, w)=0$ near $\left(\lambda_{1}, 0,0, \phi_{1}\right)$ in $M$ can be written as the form $(a, c(a), u(a), w(a))$ such that

$$
\left.\frac{d}{d a}(c(a), u(a), w(a))\right|_{a=\lambda_{1}}=-K^{-1}\left(H_{a}\left(\lambda_{1}, 0,0, \phi_{1}\right)\right)=\left(0, k_{1} \phi_{1}, \psi_{1}\right),
$$

where $k_{1}=\int_{\Omega} \phi_{1}^{2} d x /\left(2 b \int_{\Omega} \phi_{1}^{3} d x\right)>0$, and $\psi_{1} \in X_{2}$ satisfies $\Delta \psi+\lambda_{1} \psi=2 b k_{1} \phi_{1}^{2}-$ $\phi_{1}$. This is calculated using the proof of surjectivity. In particular, there exists $\delta_{5}>0$ such that for each $a \in\left(\lambda_{1}, \lambda_{1}+\delta_{5}\right), H=0$ has a unique solution in $M$ with the form $\left(a, o\left(\left|a-\lambda_{1}\right|\right),\left(a-\lambda_{1}\right) k_{1} \phi_{1}+o\left(\left|a-\lambda_{1}\right|\right), \phi_{1}+\left(a-\lambda_{1}\right) \psi_{1}+o\left(\left|a-\lambda_{1}\right|\right)\right)$. Notice that $u(a)>0$ and $w(a)>0$.

Lemma 4.3. Define

$$
O_{\delta}=\left\{(c, u) \in \mathbf{R} \times X: 0 \leq c \leq \delta,\|u\|_{W^{2,2}(\Omega)} \leq \delta\right\} .
$$

Then for any small $\delta>0$, there exists $\eta=\eta(\delta)>0$ such that, when $a \in\left(\lambda_{1}, \lambda_{1}+\eta\right)$,

1. if $(c, u)$ is a solution of (1.1) satisfying $c \geq 0$ and $u \geq 0$, then $(c, u) \in O_{\delta}$;

2. if $(c, u) \in O_{\delta}$ is a solution of (1.1), then $u \geq 0$.

Proof. The first statement can be obtained from Propositions 2.7 and 2.8 Since $c \geq 0$ and $u \geq 0$, then for some $C>0$

$$
|c|+\|u\|_{W^{2,2}(\Omega)} \leq C\left(a-\lambda_{1}\right) .
$$

For the second statement, we prove that when $a \in\left(\lambda_{1}, \lambda_{1}+\eta\right)$ for small enough $\eta>0$, any solution $(c, u) \in O_{\delta}$ satisfies

$$
\begin{aligned}
& u=\alpha \phi_{1}+v, \alpha=\int_{\Omega} u \phi_{1} d x>0,\|v\|_{W^{2,2}(\Omega)}=o(\alpha), \\
& c=o(\alpha), \text { as } \eta \rightarrow 0 .
\end{aligned}
$$

First, from (2.15), we have

$$
\alpha\left(a-\lambda_{1}\right)=\left(a-\lambda_{1}\right) \int_{\Omega} u \phi_{1} d x>c \int_{\Omega} h \phi_{1} d x .
$$

Thus if $a>\lambda_{1}$, then any solution $(c, u) \in O_{\delta}$ of (1.1) satisfies $\alpha=\int_{\Omega} u \phi_{1} d x>0$ and $c<C \alpha\left(a-\lambda_{1}\right)$. In particular, $c=o(\alpha)$ as $\eta \rightarrow 0$. The smallness of $v$ can be proved modifying an argument by Crandall and Rabinowitz CR1. In fact, when $c=0$, we can directly apply Lemma 1.12 on pages $326-327$ of [CR1], where the following (in our context) is proved:

$$
\|v\|_{W^{2,2}(\Omega)}+|\alpha| \cdot\left|a-\lambda_{1}\right| \leq|\alpha| g(\alpha),
$$

where $(a, u)=\left(a, \alpha \phi_{1}+v\right) \in V$, a neighborhood of $\left(\lambda_{1}, 0\right)$, and $g(\cdot)$ is a continuous function on $\mathbf{R}$ such that $g(0)=0$. When $c \neq 0$, we can follow the proof in pages 326-327 of [CR1] to get

$$
\begin{gathered}
\|v\|_{W^{2,2}(\Omega)}+|\alpha| \cdot\left|a-\lambda_{1}\right| \leq|\alpha| g(\alpha)+|c| \cdot\|h\|_{L^{2}(\Omega)} \\
\leq|\alpha| g(\alpha)+C|\alpha| \cdot\left|a-\lambda_{1}\right|
\end{gathered}
$$

which implies the estimate for $v$ in (4.19).

Since $\phi_{1}$ satisfies $\phi_{1}>0$ on $\Omega$ and $\partial_{n} \phi_{1}<0$ on $\partial \Omega$, then $u \geq 0$ when $\eta>0$ is small enough. 
Proof of Theorem 4.1. Fix a small $\delta>0$. Then from Lemmas 4.2 and 4.3, there exists $\eta>0$ such that for $a \in\left(\lambda_{1}, \lambda_{1}+\eta\right)$, (1.1) has a unique degenerate solution $(c(a), u(a))$ in $O_{\delta}$, all solutions in $O_{\delta}$ are nonnegative, and all nonnegative solutions are in $O_{\delta}$.

By Theorem 2.5, (1.1) has exactly two nonnegative solutions, $(0,0)$ and $\left(0, v_{a}\right)$, in $O_{\delta}$ when $c=0$. We denote the degenerate solution by $\left(c_{2}, u_{*}\right)$, and $w(a)=w_{*}$. At $\left(c_{2}, u_{*}\right)$, we verify that Theorem 2.4 can be applied here. In fact, 0 is a simple eigenvalue of (2.11) from the uniqueness of a solution to $H=0$, and $F_{c}\left(c_{2}, u_{*}\right)=$ $-h(x) \notin R\left(F_{u}\left(c_{2}, u_{*}\right)\right)$ since $\int_{\Omega}(-h(x)) w_{*} d x \neq 0$. Therefore, by Theorem 2.4 the solution set of (1.1) near $\left(c_{2}, u_{*}\right)$ can be written as a form $(c(s), u(s))$ for $s \in\left(-\delta_{7}, \delta_{7}\right)$ for some $\delta_{7}>0$, such that $c(0)=c_{2}, u(s)=u_{*}+s w_{*}+o(|s|)$, $c^{\prime}(0)=0$ and

$$
c^{\prime \prime}(0)=-\frac{2 b \int_{\Omega} w_{*}^{3} d x}{\int_{\Omega} h w_{*} d x}<0
$$

from (2.7). Thus the branch of solutions turns to the left at $\left(c_{2}, u_{*}\right)$. We call the subbranch containing $(c(s), u(s))$ with $s>0$ the upper branch, and the one containing $(c(s), u(s))$ with $s<0$ the lower branch. Both branches continue to the left up to $c=0$ without any more turnings, since $\left(c_{2}, u_{*}\right)$ is the unique degenerate solution, and the solutions on both branches are nonnegative by Lemma 4.3. The upper branch must be coincident to the branch of maximal solutions which we obtain in Theorem 3.2 In fact, the branch of maximal solutions emanates from $(c, u)=$ $\left(0, v_{a}\right)$, continues as $c$ increases until it reaches a degenerate solution $\left(c^{*}, u^{*}\right)$, which is nonnegative as a limit of (positive) maximal solutions, thus $\left(c^{*}, u^{*}\right) \in O_{\delta}$, and it must be coincident to $\left(c_{2}, u_{*}\right)$. The lower branch must meet $(0,0)$ when $c=0$.

All solutions in $O_{\delta}$ are positive except $(0,0)$, since they have the form $u=$ $\alpha \phi_{1}+v$ with $v$ small (see the proof of Lemma 4.3). There is no other nonnegative solution of (1.1), since all nonnegative solutions must lie in $O_{\delta}$. The solutions on the upper branch are stable from Theorem 3.2. The solutions on the lower branch are nondegenerate, and they have Morse index 1 near the turning point or $(0,0)$; hence each solution on the lower branch has Morse index 1 by the continuity.

\section{REFERENCES}

[AB] Afrouzi, G. A.; Brown, K. J., On a diffusive logistic equation. J. Math. Anal. Appl., 225, (1998), no. 1, 326-339. MR 99g:35130

[ACS] Ali, Ismael; Castro, Alfonso; Shivaji, R., Uniqueness and stability of nonnegative solutions for semipositone problems in a ball. Proc. Amer. Math. Soc., 117, (1993), no. 3, 775-782. MR 93d:35048

$[\mathrm{ABC}]$ Ambrosetti, Antonio; Brézis, Haim; Cerami, Giovanna, Combined effects of concave and convex nonlinearities in some elliptic problems. J. Funct. Anal., 122, (1994), no. 2, 519543. MR 95g:35059

[BC] Brauer, Fred; Castillo-Chávez, Carlos, Mathematical models in population biology and epidemiology. Texts in Applied Mathematics, 40. Springer-Verlag, New York, (2001). CMP 1822695

[BK] Brezis, H.; Kamin, S, Sublinear elliptic equations in $\mathbf{R}^{n}$, Manu. Math., 74, (1992), 87-106. MR 93f:35062

[CMS] Castro, Alfonso; Maya, C.; Shivaji, R., Nonlinear eigenvalue problems with semipositone structure. Elec. Jour. Differential Equations, Conf. 5., (2000), 33-49. CMP 1799043

[CS] Castro, Alfonso; Shivaji, R., Positive solutions for a concave semipositone Dirichlet problem. Nonlinear Anal., 31, (1998), no. 1-2, 91-98. MR 98j:35061 
[CC1] Cantrell, Robert Stephen; Cosner, Chris, Diffusive logistic equations with indefinite weights: population models in disrupted environments. Proc. Roy. Soc. Edinburgh Sect. A, 112, (1989), no. 3-4, 293-318. MR 91b:92015

[CC2] Cantrell, R. S.; Cosner, C., The effects of spatial heterogeneity in population dynamics. J. Math. Biol., 29, (1991), no. 4, 315-338. MR 92b:92049

[Cl] Clark, Colin W., Mathematical Bioeconomics, The Optimal Management of Renewable Resources, John Wiley \& Sons, Inc. New York (1990). MR 91c:90037

[CP] Clément, Ph.; Peletier, L. A., An anti-maximum principle for second-order elliptic operators. J. Differential Equations, 34, (1979), no. 2, 218-229. MR 83c:35034

[CR1] Crandall, Michael G.; Rabinowitz, Paul H, Bifurcation from simple eigenvalues. J. Functional Analysis, 8, (1971), 321-340. MR 44:5836

[CR2] Crandall, Michael G.; Rabinowitz, Paul H., Bifurcation, perturbation of simple eigenvalues and linearized stability. Arch. Rational Mech. Anal., 52, (1973), 161-180. MR 49:5962

[F] Fisher, R.A., The wave of advance of advantageous genes. Ann. Eugenics, 7, (1937), 353369.

[He] Henry, Daniel, Geometric theory of semilinear parabolic equations. Lecture Notes in Mathematics, 840. Springer-Verlag, Berlin-New York, (1981). MR 83j:35084

[Ho] Holling, C.S., The components of predation as revealed by a study of small-mammal predation on the European pine sawfly. Canadian Entomologist, 91, (1959), 294-320.

[KPP] Kolmogoroff, A., Petrovsky, I, Piscounoff, N, Study of the diffusion equation with growth of the quantity of matter and its application to a biological problem. (French) Moscow Univ. Bull. Math. 1, (1937), 1-25.

[KS] Korman, Philip; Shi, Junping, New exact multiplicity results with an application to a population model, Proceedings of Royal Society of Edinburgh Sect. A, 131, (2001), 11671182. CMP 1862448

[M] Murray, J. D., Mathematical biology. Second edition. Biomathematics, 19., SpringerVerlag, Berlin, (1993). MR 94j:92002

[OS] Ouyang Tiancheng; Shi, Junping, Exact multiplicity of positive solutions for a class of semilinear problems: II. Jour. Differential Equations, 158, 94-151, no. 1, (1999). MR 2001b:35117

[Sa] Sattinger, D. H., Monotone methods in nonlinear elliptic and parabolic boundary value problems. Indiana Univ. Math. J., 21, (1971/72), 979-1000. MR 45:8969

[S] Shi, Junping, Persistence and bifurcation of degenerate solutions. Jour. Func. Anal., 169, (1999), no. 2, 494-531. MR 2001h:47115

[SS] Shi, Junping; Shivaji, Ratnasingham, Global bifurcation for concave semipositon problems, Recent Advances in Evolution Equations, (2002). (to appear)

[SY] Shi, Junping; Yao, Miaoxin, On a singular nonlinear semilinear elliptic problem. Proc. Roy. Soc. Edingbergh Sect. A, 128, (1998), no. 6, 1389-1401. MR 99j:35070

[Y] Yosida, Kosaku, Functional analysis. Fifth edition. Grundlehren der Mathematischen Wissenschaften, Band 123. Springer-Verlag, Berlin-New York, (1978). MR 58:17765

Department of Mathematics, Mississippi State University, Mississippi State, MissisSIPPI 39762

E-mail address: so1@ra.msstate.edu

Department of Mathematics, College of William and Mary, Williamsburg, Virginia 23187 and Department of Mathematics, Harbin Normal University, Harbin, Heilongjiang, P. R. China 150080

E-mail address: shij@math.wm.edu

Department of Mathematics, Mississippi State University, Mississippi State, MissisSIPPI 39762

E-mail address: shivaji@ra.msstate.edu 